\title{
COMERCIO MUNDIAL DE BIENES Y SERVICIOS AMBIENTALES. DESEMPEÑO Y RETOS DE LA INDUSTRIA MEXICANA
}

\author{
René Lara* \\ Petr Sauer** \\ Ludmila Sterbová ${ }^{* * *}$
}

- $\mathrm{n}$ el marco de la globalización, el comercio internacional es una
de las principales actividades y se supone que beneficia a todas las partes. Desde los años ochenta, la política económica mexicana ha experimentado grandes cambios profundos. Aunque algunos han llevado a descubrir nuevas capacidades, se han hecho evidentes los problemas ocasionados por las nuevas políticas. México pasó de ser una economía cerrada, con una rígida intervención estatal, a una economía de mercado abierto con poca intervención y un nuevo marco institucional. En Iberoamérica se dio libre acceso a los mercados, bajo la premisa de que un Estado de gran tamaño es incapaz de organizar la economía e ignora la complejidad social, económica y política de la región (Guerrero, 1994). El menosprecio del sector público y de su intervención estuvo acompañado de la obsesión por la privatización y la promesa de altas tasas de crecimiento (Castrillón, 2013).

La nueva economía mexicana se caracteriza por una estructura industrial formada por pequeñas y medianas empresas (Pymes), que aumentaron la formación bruta de capital, elevaron la proporción de los servicios en el PIB y concentraron los flujos de comercio hacia la industria del norte de América a partir de los años noventa (Muñoz, 2005), cuando el nuevo modelo de desarrollo sustituyó la propiedad

*Universidad de Economía, Praga, República Checa, [1ararene83@yahoo.com.mx]. ** Universidad de Economía, Praga, República Checa, [sauer@vse.cz].

*** Universidad de Economía, Praga, República Checa, [stervobl@vse.cz]. Este artículo es resultado del proyecto IGA n. ${ }^{\circ}$ F2/26/2014, Impact of economic liberalization on environmental policy. Fecha de recepción: 19-01-2017, fecha de modificación: 24-05-2017, fecha de aceptación: 04-09-2017. Sugerencia de citación: Lara, R; Sauer, P. y Sterbová, L. (2017). ¿Comercio mundal de bienes y servicios ambientales. Desempeño y retos de la industria mexicana, Revista de Economía Institucional 19(37), 185-200. Dor: https://doi.org/10.18601/01245996.v19n37.10 
pública y la regulación por la propiedad privada y el libre mercado, y redujo la protección para atraer inversión extranjera y fomentar el comercio internacional (Lustig, 2001).

La entrada al GATT en 1986 dio inicio a una reestructuración económica y la apertura de mercados se convirtió en el objetivo principal de la agenda (Morales, 1997), para explotar las ganancias económicas del comercio con otros países, en especial con Estados Unidos. E1 Tratado de Libre Comercio de América del Norte (TLCAN), que entró en vigencia en 1994, fue un cambio radical que puso fin definitivo al modelo de sustitución de importaciones (Hanson, 2010).

En vez de favorecerlos, las reformas tuvieron efectos negativos en diversos sectores; sobre todo debido a los vacíos institucionales creados por el inadecuado diseño de política, que excluyó a la mayoría de las Pymes (Lustig, 2001). Así, los beneficios prometidos de la liberalización quedaron fuera del alcance de gran parte de la industria y de los empresarios. En las negociaciones del TLCAN se incluyó la protección ambiental y sus objetivos parecían claros, pero no los mecanismos para lograrlos (Ranger, 1993); además, México es miembro de la OMc desde 1995, una de cuyas prioridades es reducir o eliminar las tarifas de bienes y servicios asociados a la protección ambiental. Y en el tema del desarrollo sostenible no hay congruencia entre el discurso y la acción, pues responde más a la presión internacional que a una iniciativa propia (Ahumada et al., 2012).

Este artículo analiza la estrategia de liberalización del comercio de bienes y servicios ambientales (BysA), y las consecuencias para México del comercio con países -con una industria ambiental madura y mercados saturados- que buscan exportar sus excedentes. La primera sección repasa la historia de la liberalización del comercio y comenta sus consecuencias para la industria mexicana. La segunda sección aborda el debate sobre los beneficios de la liberalización de bienes y servicios ambientales y la complejidad de definirlos y elaborar listas de esos bienes. La tercera sección describe el estado actual de la industria ambiental mexicana, su oferta y sus limitaciones. La cuarta sección evalúa sus principales segmentos en el TLCAN, y la contribución de la industria mexicana a su competitividad. La quinta sección examina los factores de demanda para entender los efectos ocasionados de la política económica y comercial en el desarrollo de la industria. En la sección final se presentan las conclusiones. 


\section{LIBERALIZACIÓN DEL COMERCIO Y LA INDUSTRIA MEXICANA}

Con el ingreso al gatt, México dio a sus socios el estatus de "nación más favorecida", a fin de aumentar el comercio libre de aranceles, y con el TLCAN se esperaba avanzar en ese objetivo dada la importancia del mercado estadounidense. Con la apertura comercial las exportaciones aumentaron un $16,5 \%$ anual y las importaciones un $11,5 \%$ en el periodo 1994-1998; en el año 2000, el 92\% del comercio internacional de México se realizaba con países industrializados; Estados Unidos recibía el $85 \%$ de las exportaciones, lo que reforzó su importancia (Puyana, 2003). También cambió el tipo de bienes exportados, de recursos naturales y materias primas a productos manufacturados con alta tecnología, debido a la modificación de las prioridades de las grandes empresas nacionales y extranjeras que operan en México y a la desregulación de la economía después del ingreso al gATT (Morales, 1997). Una característica de esta especialización es la importancia del capital extranjero en las grandes empresas que concentran el comercio mexicano. En 1994 había 573 empresas, el 2,7\% del total, que aportaban el $80 \%$ de las exportaciones no petroleras y el $20 \%$ provenía de 20.846 empresas, el 97,3\% restante. Si bien el número de empresas exportadoras aumentó en un 67\% entre 1993 y 1999 , llegó a más de 30 mil, la mayoría eran pequeñas; en 1994 las pequeñas empresas representaban el $74 \%$ de la industria, las medianas el 15\% y las grandes el $11 \%$.

Las grandes empresas con participación de capital extranjero controlan la producción industrial y son el núcleo del comercio internacional, que en su mayoría es de carácter intersectorial, un 62\% del total en 1998 (Puyana, 2003). El comercio intersectorial es característico de mercados jerárquicos que responden a divisiones funcionales de la producción entre las principales industrias del país de origen y sus asociados en el extranjero, lo que, junto con el marco institucional del TLCAN sobre inversiones y acceso a mercados, benefició sobre todo a las industrias de bienes manufacturados de alta tecnología, que complementaban la producción de firmas en el extranjero. Este tipo de comercio impide el desarrollo de una ventaja comparativa, pues la mayoría de la inversión extranjera directa (IED) de Estados Unidos busca complementar la industria de ese país. Así, la industria mexicana tiene pocas posibilidades de cubrir sus necesidades nacionales y pierde competitividad en los demás mercados internacionales (Kehoe y Ruhl, 2011). Además, las empresas mexicanas están en desventaja porque tienen menos conocimientos y recursos para resolver disputas legales con firmas más grandes (Morales, 1997). Dada la 
importancia del mercado estadounidense y su carácter jerárquico es necesario examinar sus efectos en el sector exportador mexicano, en el contexto del TLCAN.

\section{BENEFICIOS DE LA LIBERALIZACIÓN DE BIENES AMBIENTALES}

En la Cuarta Conferencia Ministerial de la omc, realizada en Doha en noviembre de 2001, se acordó negociar la reducción o eliminación de aranceles y disminuir las barreras no relacionadas con el comercio en вysA, una estrategia que beneficiara a todos los socios comerciales. Esa estrategia buscaba aumentar el flujo de comercio de los países desarrollados a nuevos mercados, sobre todo a países en desarrollo, que se beneficiarían con una mejor calidad ambiental y el cumplimiento de los estándares internacionales de exportación (Vikhlyaev, 2004). La liberalización comercial de bienes ambientales impulsaría el desarrollo sostenible y el desarrollo económico, al mejorar el acceso a tecnología, conocimientos e insumos sustitutos menos perjudiciales para el medio ambiente, a un menor costo (Mathew y Fernández, 2009). Aunque estos autores tienen en cuenta las barreras que impiden la liberalización total -la más importante de las cuales es quizá la brecha entre las condiciones económicas de los países desarrollados y en desarrollo-, se preguntan si el objetivo de la liberalización es impulsar la competencia en los mercados o competir por ellos. Los mercados de los países desarrollados están saturados y sus consumidores son sofisticados, lo que reduce las ventas de Bysa en algunos segmentos; además, allí la regulación ambiental tiene un gran peso en la variación de la demanda. Aunque quizá lo más relevante sea la saturación de los mercados, pues induce a buscar nuevos destinos para vender los excedentes, sobre todo los de Europa Occidental, Estados Unidos y Japón, que copan el 85\% del mercado de вysA.

Si bien en los países en desarrollo hay sólidos factores de demanda, como el aumento de la población y la urbanización, sus mercados de bienes ambientales son menos homogéneos y la industria es temprana, lo que obstaculiza la liberalización. Primero, los aranceles a la importación son más elevados y dificultan la liberalización aunque sea posible iniciar con reducciones arancelaras para establecer condiciones mínimas de integración. Pero esto los lleva a una encrucijada: al no poder competir en el mercado global se inclinan al proteccionismo. Segundo, surgen barreras no relacionadas con el comercio por diferencias en la regulación ambiental, pues la falta de uniformidad de las normas afecta principalmente a los países en desarrollo que suelen tener una ventaja comparativa en productos ambientalmente prefe- 
ribles (PAP), que pueden requerir alguna certificación para ingresar en algunos mercados. Tercero, el costo de transferir conocimientos requiere importar personal capacitado para difundirlos. Por último, en los países en desarrollo el gobierno suele ser el único comprador de Bysa, pues es el responsable de proporcionar la mayoría de los servicios públicos.

La definición de los Bysa exige especial atención ya que delimita el tamaño de la industria ambiental. Hoy existe un intenso debate sobre la manera de definirlos. Por ejemplo, se considera que son tecnologías, productos manufacturados y químicos utilizados para proporcionar servicios ambientales; que incluyen una amplia gama de artículos, incluidos los PAP como sustitutos de productos similares (Sang y Jisun, 2011). Al proponer definiciones es necesario considerar el uso dual de algunos productos, el cual justifica que entren en dicha categoría. Por ejemplo, los tubos se pueden usar en la construcción de una turbina eólica o de una planta que funcione con carbón. En las negociaciones, el uso dual es un obstáculo para mejorar la especificidad del Sistema Armonizado de Códigos Arancelarios dada la falta de claridad en la tipificación de los bienes ambientales.

Las definiciones y listas de Bysa difieren según la organización y los participantes. La UNCTAD (2003) los define como entrega de servicios ambientales y productos más ecológicos; la (OCDE, 1999) basa su definición en la medición, prevención, limitación, minimización y corrección del daño ambiental. Aunque quizá las definiciones más caracterizadas son las del Foro de Cooperación Económica AsiaPacífico (APEC) y de la Organización Mundial de Comercio elaborada con base en la de la APEC y la OCDE, esta incluye una lista central de 26 artículos y ha recibido propuestas de algunos miembros (Hamwey et al., 2003). Estas listas abarcan bienes de capital, lo que es una ventaja para los países desarrollados que son líderes en el mercado y ha llevado a numerosos países en desarrollo a ser importadores netos de BysA, como se manifestó en 1996, cuando la industria ambiental tuvo un boom y los países desarrollados acapararon las ganancias, mientras que algunos países de Asia aumentaron la exportación de BySA en un 75\% (Vhyklyaev, 2004).

En suma, las diferencias entre países desarrollados y en desarrollo afectan las definiciones y listas de bienes que delimitan el tamaño de la industria ambiental y sus segmentos de mercado. Aunque existe consenso sobre los beneficios de la liberalización del comercio de estos productos, hay escasos avances globales en la apertura de sus mercados (Znamenacková et al., 2014). 


\section{LA INDUSTRIA AMBIENTAL MEXICANA}

La definición de Bysa ayuda a entender cómo está conformada la industria ambiental. La definición de la OCDE es la más conveniente para clasificar los bienes producidos en México porque es exhaustiva y contiene equivalencias de los productos del Sistema Armonizado, lo que facilita cuantificar su volumen de comercio (Muñoz, 2005). Este autor, que clasificó los bienes desagregando datos de Cuentas Nacionales y resultados de un censo económico, estableció tres categorías: el grupo A engloba bienes y servicios para control de la contaminación ligados a las políticas de reducción del desequilibrio ambiental a través de instrumentos de mando y control. El grupo B cubre bienes y servicios asociados a tecnologías más limpias así como bienes intermedios y finales ligados a las políticas para mejorar la eficiencia ecológica del sistema productivo y el consumo. El grupo $\mathrm{C}$ se compone de bienes y servicios vinculados al uso racional de los recursos naturales, ligados a políticas de manejo de esos recursos.

En México existen 6.368 firmas que encajan en alguna de las categorías anteriores, pero ese número es muy pequeño, solo representa el 1,15\% del total. La producción de bienes ambientales se concentra en el segmento de control de la contaminación; después vienen las empresas que administran recursos naturales, sobre todo el abastecimiento de agua; en tercer lugar se encuentra el segmento que produce PAP. La importancia del sector de servicios ambientales ha aumentado con la expansión de los servicios directos, complemento de los bienes ambientales, y de los servicios indirectos, para distribución y mantenimiento de los bienes ambientales. Cabe destacar que el número de empresas del sector se ve afectado por las diferencias internas en la política ambiental, y que el mercado interno de вysA es relativamente pequeño, aunque con gran potencial de crecimiento por el dinamismo de algunos segmentos.

En general, la industria ambiental mexicana está poco desarrollada y el tamaño de sus empresas es un freno para el crecimiento. Si bien la omc señaló que luego de la crisis financiera de 1994-1995 aumentó la producción de BysA, a una tasa anual del 4.4\% en 1998, también aumentó el número de empresas pero su tamaño se redujo. En 1993, el promedio de empleados por empresa era de 42 y en 1998 bajó a 34; esta disminución del empleo se complementó con salarios bajos para aumentar la competitividad. Así, la estrategia de la competitividad se basó en una reducción de los costos de producción que precarizó el empleo en vez modernizar las empresas a través de inversiones. E1 sector financiero es importante para el crecimiento 
económico, pero en México no aporta recursos para actividades con alto potencial productivo, como la industria ambiental, que como se verá poco participa en el sector más rentable de BySA en la región del TLCAN (Ramírez, 2011).

Las ventas se concentran en bienes para el control de la contaminación (grupo A) y bienes para administración de recursos naturales (grupo C), debido principalmente al desarrollo histórico de la industria y la liberalización de la economía y al aumento de la competencia asociada a la mayor liberalización del comercio. En 2001 había 807 empresas de servicios ambientales, un $27 \%$ más que en el año anterior; estas firmas servían a varios segmentos del mercado.

Para este estudio se intentó obtener información del desempeño de la industria para hacer estimaciones propias, pero los problemas de definición y las deficientes bases de datos dificultaron la recopilación, por lo que tuvimos que recurrir a información de diversos autores para esclarecer el panorama de la industria, que en 1996 aportó el 0,44\% del pib y en 2006 el 0,59\%, con un bajo número de empresas de pequeño tamaño (Ferrier, 2010). El cuadro 1 muestra su distribución.

Cuadro 1

La industria ambiental en México, 2006

\begin{tabular}{lc}
\hline \multicolumn{1}{c}{ Segmento } & $\begin{array}{c}\text { Número estimado } \\
\text { de compañías }\end{array}$ \\
\hline Equipo & 200 \\
Equipo y químicos para agua & 100 \\
Control de la contaminación del aire & 30 \\
Instrumentos y sistemas de información & 300 \\
Equipo para manejo de residuos & 30 \\
Tecnología para prevención y procesos & \\
Servicios & 1.200 \\
Manejo de residuos sólidos* & 350 \\
Manejo de residuos peligrosos* & 900 \\
Consultoría e ingeniería & 120 \\
Servicios industriales y de remediación & 70 \\
Servicios analíticos & 2.340 \\
Trabajos de tratamiento de agua* & \\
Recursos & 1.360 \\
Servicio de abastecimiento de agua & 1.200 \\
Recuperación de recursos & 100 \\
Sistemas de energía y poder limpios & 8.300 \\
Total &
\end{tabular}

* Incluye entidades que generan ingreso o prestan servicios en el sector público; sobre todo de agua, aguas residuales y manejo de residuos.

Fuente: Ferrier $(2010,5)$.

Estos datos son muy dicientes frente a los de la industria estadounidense, el principal socio de México en la región del trcan. Estados Unidos es uno de los mayores productores de Bysa en el mundo, con cerca de 117 mil empresas en el sector de tecnologías ambientales, 
cuya industria ha generado ingresos por 196,5 billones de dólares y creado 1,4 millones de empleos en el país. Su mercado nacional es grande, y aunque solo algunas empresas exportan sus productos, compiten fuertemente con las de Alemania y Japón por otros mercados, especialmente de países en desarrollo (Avery y Boadu, 2004). E1 cuadro 2 muestra la participación y las ganancias de los actores clave en el comercio internacional de bienes ambientales.

México enfrenta grandes dificultades que mantienen estancada su industria ambiental, sobre todo porque no ha dedicado los recursos necesarios para mejorar su infraestructura ambiental. Desde los años ochenta el país ha experimentado crisis económicas recurrentes, que sumadas a la devaluación de la moneda en 1994, cuando se implementó el TLCAN, redujeron drásticamente el presupuesto y duplicaron los precios de la tecnología ambiental (Feagans, 1997). En un estudio sobre la transición tecnológica de tres empresas mexicanas, los autores encontraron que los choques económicos redujeron sustancialmente la inversión en investigación y desarrollo, lo que restringió la producción de bienes con alto valor agregado (Dutrénit et al., 2003). Otra dificultad para adoptar tecnologías limpias es que los productores tienen poco conocimiento de tecnologías más eficientes y escasos recursos para invertir en el cambio de procesos.

Cuadro 2

Actores clave en el comercio internacional de bienes ambientales ${ }^{1}$ (Millones de dólares)

\begin{tabular}{|c|c|c|c|c|}
\hline País & Exportaciones & $\begin{array}{l}\text { Participación en el } \\
\text { comercio mundial }\end{array}$ & Importaciones & $\begin{array}{l}\text { Participación en el } \\
\text { comercio mundial }\end{array}$ \\
\hline Unión Europea & $34.248,0$ & 19,3 & $28.802,2$ & 16,3 \\
\hline Japón & $22.842,5$ & 12,9 & $5.170,8$ & 3,0 \\
\hline Estados Unidos & $17.651,9$ & 10,0 & $21.228,9$ & 12,0 \\
\hline China & $21.813,2$ & 12,3 & $19.552,7$ & 11,1 \\
\hline Corea & $6.786,0$ & 3,9 & $11.068,1$ & 6,4 \\
\hline México & $4.811,7$ & 2.8 & $3.327,9$ & 1,9 \\
\hline Total mundial & $177.187,1$ & & $176.877,5$ & \\
\hline
\end{tabular}

Para enfrentar esta situación se decidió fortalecer la regulación ambiental y suprimir los aranceles a la importación de tecnologías ambientales en el marco del TLCAN, asegurando así una oferta suficiente. Además, México obtuvo apoyo financiero de Japón, del BID y del Banco Mundial para financiar programas de limpieza y recuperar el mercado nacional de BysA dado que solo era posible tratar el 10\% de las aguas residuales municipales y solo el 16\% de los residuos peligrosos. Apenas se recolectaba el 70\% de la basura. 
Un problema adicional es que, en tiempos de crisis, México no separó las reformas nacionales de las medidas tomadas en conjunto con sus socios extranjeros, y las crisis se asocian a pérdida de soberanía (Konvitz, 2012). Además, durante la crisis de 1994-1995, el gasto en infraestructura cayó por la escasa disponibilidad de crédito. Konvitz muestra que los diferentes niveles de gobierno conseguían financiación para proyectos que concluyeran durante su periodo administrativo, y dejar la deuda a sus sucesores. Esto indujo a las entidades financieras a reducir la oferta de créditos, y los recursos invertidos en infraestructura no siempre favorecieron el desarrollo económico, social y ambiental. En este contexto, el segmento de servicios ambientales se expandió, pero el grueso de la industria se rezagó, como muestra el balance comercial de BysA.

\section{EL COMERCIO DE BYSA EN EL TLCAN}

Aunque es difícil obtener los datos de aranceles para analizar el impacto del comercio de вysa en la región, las cifras de exportaciones e importaciones dan indicios útiles. De 1995 a 2005 se duplicó el mercado de bienes ambientales en la región (Zhang, 2010). A esto se debe la importancia del mercado mexicano para la industria ambiental estadounidense. México es el mercado más grande de las Américas, cuyas ventas eran de unos 3 billones de dólares en 1997 y se proyectaba que pasarían a 3,8 billones en 2008 .

Cuadro 3

Comercio de bienes y servicios ambientales de México en el tLCAN, 2001* (Millones de dólares)

\begin{tabular}{|c|c|c|c|c|}
\hline Tipo de bienes & $\begin{array}{l}\text { Exportaciones a } \\
\text { Estados Unidos }\end{array}$ & $\begin{array}{l}\text { Importaciones de } \\
\text { Estados Unidos }\end{array}$ & $\begin{array}{c}\text { Exportaciones a } \\
\text { Canadá }\end{array}$ & $\begin{array}{c}\text { Importaciones de } \\
\text { Canadá }\end{array}$ \\
\hline \multicolumn{5}{|l|}{ Equipo } \\
\hline Equipo y químicos para agua & 1,3 & 300,5 & 0,1 & 4,4 \\
\hline $\begin{array}{l}\text { Equipo de control } \\
\text { para la contaminación atmosférica }\end{array}$ & 1,3 & 98,4 & 0,1 & 4,5 \\
\hline Instrumentos y sistemas de información & 0,00 & 35,0 & 0,00 & 0,2 \\
\hline Equipo para manejo de residuos & 10,5 & 62,1 & 1,6 & 4,3 \\
\hline Tecnologías de proceso y prevención & 0,2 & 3,2 & 0,0 & 0,0 \\
\hline \multicolumn{5}{|l|}{ Servicios } \\
\hline Manejo de residuos sólidos & 3,3 & 40,8 & 0,0 & 0,6 \\
\hline Manejo de residuos peligrosos & 0,4 & 31,8 & 0,0 & 0,1 \\
\hline Consultoría e ingeniería & 0,5 & 58,7 & 0,1 & 0,6 \\
\hline $\begin{array}{l}\text { Servicios industriales } \\
\text { y de saneamiento }\end{array}$ & 0,7 & 23,8 & 0,3 & 0,9 \\
\hline Servicios analíticos & 0,0 & 3,7 & 0,0 & 0,2 \\
\hline Tratamiento de agua & 5,4 & 46,9 & 0,0 & 0,1 \\
\hline \multicolumn{5}{|l|}{ Recursos } \\
\hline Empresas públicas para manejo de agua & 0,3 & 26,1 & 0,0 & 0,1 \\
\hline Recuperación de recursos & 0,3 & 142,2 & 0,1 & 1,2 \\
\hline Sistemas y servicios de energía limpia & 1,1 & 41,1 & 0,0 & 0,3 \\
\hline Agricultura sostenible & 32,1 & 4,3 & 4,5 & 1,3 \\
\hline Silvicultura sostenible & 5,6 & 0,0 & 0,7 & 0,0 \\
\hline Ecoturismo & 53,6 & 0,1 & 5,4 & 0,3 \\
\hline Total & 116,6 & 918,7 & 12,8 & 19,0 \\
\hline
\end{tabular}

* Según la definición de la ocDE (1999).

Fuente: Comisión para la Cooperación Ambiental (2004, 10-12). 
E1 mercado mexicano es un destino estratégico para los países exportadores de BysA, y se estimaba que el mayor aumento de la demanda de exportaciones de la industria ambiental estadounidense provendría de países en desarrollo, incluido México (Avery y Boadu, 2004). El balance comercial de México entre 1993 y 1998 muestra el sesgo importador: 40 mil millones de dólares en mercancías importadas y solo 21 mil millones exportadas, a pesar del aumento de la tasa de exportaciones (Muñoz, 2005). El cuadro 3 muestra las exportaciones y las importaciones de BySA de cada sector de la industria mexicana a sus socios del trcan. Según estas cifras, México es más competitivo en ecoturismo, agricultura y silvicultura sostenibles; pero es necesario analizar la participación de cada categoría en el valor total del comercio de la región para determinar cuáles son los segmentos clave; esto se muestra en el cuadro 4.

Cuadro 4

Participación de cada sector en el comercio de la región, 2001* (Millones de dólares)

\begin{tabular}{lrc}
\hline Tipo de bienes & Comercio & $\begin{array}{c}\text { Participación } \\
(\%)\end{array}$ \\
\hline Equipo & 67 \\
Equipo y químicos para agua & $1.469,8$ & \\
Equipo de control para la contaminación atmosférica & 590,7 & \\
Instrumentos y sistemas de información & 123,2 & \\
Equipo para manejo de residuos & 535,8 & 17 \\
Tecnologías de proceso y prevención & 11,7 & \\
Servicios & 130,6 & \\
Manejo de residuos sólidos & 48,7 & \\
Manejo de residuos peligrosos & 372,1 & \\
Consultoría e ingeniería & 63,6 & \\
Servicios industriales y de saneamiento & 15,4 & \\
Servicios analíticos & 69,5 & \\
Tratamiento de agua & 42,5 & \\
Recursos & 235,0 & \\
Empresas públicas para manejo de agua & 82,2 & \\
Recuperación de recursos & 197,6 & \\
Sistemas y servicios de energía limpia & 19,5 & \\
Agricultura sostenible & 78,5 & \\
Silvicultura sostenible & $4.086,6$ & 100 \\
Ecoturismo & & \\
Total & & \\
* En la suma de las exportaciones e importaciones de los tres grupos bilaterales: Estados \\
Unidos-Canadá, Estados Unidos-México y México-Canadá. \\
Fuente: Comisión para la Cooperación Ambiental (2004, 13).
\end{tabular}

Además del sesgo importador, el país es más competitivo en las actividades que tienen menor participación en el mercado. No obstante, puede desarrollar ventajas en la producción de bienes para tratamiento de aguas residuales, manejo de residuos sólidos, manejo de energía y energías renovables, y en equipo de evaluación y monitoreo (Muñoz, 
2005). Puesto que la contribución de México en bienes de capital, rubro clave en el TLCAN, es muy pequeña, es necesario pensar cómo se podría impulsar el desarrollo de la industria para aumentar su participación en los segmentos más rentables.

\section{INCIDENCIA DE LAS POLÍTICAS EN LA DEMANDA DEL MERCADO INTERNOY EN EL COMERCIO INTERNACIONAL}

Los factores de demanda de BySA más importantes en el mercado mexicano tienen que ver con la regulación y el marco institucional, porque el crecimiento de esta industria ambiental depende de ambos (ibíd.). Otro factor importante es la presión social ante la falta de atención a los problemas ambientales y la difusión en los medios de la necesidad de conservar los recursos naturales, que ha aumentado la conciencia social. Además, la presión internacional y la globalización obligaron a México a no ignorar el tema ambiental en el comercio internacional, a usar tecnologías más limpias y cumplir los estándares internacionales (Stern, 1993). Y se suponía que las empresas nacionales buscarían desarrollar ventajas competitivas y comparativas para participar en nuevos mercados (Gallegos y Régibeau, 2008).

E1 TLCAN ha contribuido a establecer estándares ambientales más elevados en el comercio, a mejorar la regulación ambiental y a ampliar la oferta de вysA (Ranger, 1993), aunque, dado el estado de la industria y de la regulación, no ha tenido el impacto esperado en los sectores productivos, que siguen reacios a adoptar nuevos bienes ecológicos y tecnologías más limpias, bien sea por falta de recursos, de interés o de conocimientos.

Se ha propuesto entonces ligar la liberalización de вysa a las metas de desarrollo sostenible, añadiendo la dimensión social, económica y geográfica, para determinar los BysA que requiere la economía mexicana.Pero "no hay certeza de que la nueva estrategia pueda aumentar las exportaciones de la industria ambiental mexicana y menos de que coadyuve a la consolidación de una oferta doméstica suficiente" (Lendo, 2005). La liberalización de estos bienes y servicios es parte del Programa Nacional de Medio Ambiente y Recursos Naturales desde 2001 para impulsar las exportaciones del sector. Sin embargo, los resultados no han sido del todo favorables y continúa el déficit comercial de BysA. En 2006, el 44\% de la demanda nacional era cubierta por la industria local y el 56\% restante por las importaciones, lo cual muestra que se mantiene el sesgo importador.

E1 Plan Nacional de Desarrollo (PND) determina los procedimientos de participación y las directrices de política a lo largo de un sexenio. 
Se supone que se elabora en forma representativa y democrática, aunque desde los ochenta predominan el mercado, la apertura comercial y la desincorporación de las empresas públicas, de modo que los criterios económicos se superponen a los sociales (González, 2008). Esto afecta a la industria ambiental, que por sus características es perjudicada por la escasa coordinación y cohesión entre políticas, instituciones y niveles de gobierno. Aunque el PND 2007-2012 intentó mejorar la coordinación interinstitucional e intersectorial para dar sostenibilidad al desarrollo, la legislación actual no permite hacer ajustes con facilidad (Ahumada et al., 2012). Según estos autores, el PND orienta la política sectorial; pero crea vacíos normativos para integrar el medio ambiente en el proceso de planeación, pues ni la Constitución ni la Ley de Planeación contemplan un horizonte temporal adecuado para las metas de desarrollo sostenible. Solo se dispone de seis meses, después de la posesión del presidente de la república, para elaborar y publicar el PND; su vigencia no puede exceder el sexenio correspondiente y esto impide la evaluación ambiental estratégica (EAE) de las propuestas de política para garantizar que se tenga en cuenta el medio ambiente, así como consideraciones económicas y sociales en las etapas tempranas de la toma de decisiones, antes de iniciar los proyectos. México debe diseñar su propia EAE, ya que al no haber un formato internacional único se puede realizar con base en la experiencia internacional y las particularidades del país, de modo que complemente la evaluación de impacto ambiental (EIA), que si bien es el instrumento preventivo más común, no garantiza la sostenibilidad en los países en desarrollo pues se aplica después de implementar los proyectos.

La EIA sistemática para determinar el impacto ambiental de proyectos de desarrollo suscita un fuerte debate político, pues incide en una toma de decisiones informadas que tiene en cuenta el medio ambiente (Bojórquez et al., 2005). Aunque es ampliamente aceptada en el ámbito internacional y local porque es indispensable para fijar criterios de sostenibilidad, requiere mucha información para hacerla bien. En México existen diversas instituciones ambientales, como la Secretaría de Medio Ambiente y Recursos Naturales y el Instituto Nacional de Ecología, pero casi toda la información que producen es descriptiva y sus indicadores no vinculan integralmente los aspectos sociales y económicos al cambio ambiental, lo que impide evaluar la efectividad de las políticas. La Eı aún es débil para convertirse en instrumento confiable (Perevochtchikova, 2013; Tortajada, 2000).

Corregir las fallas del PND y fortalecer la EIA mejoraría el diseño de políticas, que suelen consistir en enunciados ambiguos o tienen propó- 
sitos implícitos, de modo que los objetivos son temas de investigación y no de evaluación. Esas medidas darían una base mínima para que la formulación de políticas sea congruente con su implementación, y para evaluar su éxito o su fracaso (Guerrero, 1994). Es imperativo dar un giro al diseño de políticas para que tomen en cuenta el interés público y susciten apoyo político (Lindblom, 1994).

Un paso importante para desarrollar la industria ambiental y lograr las metas de desarrollo sostenible es reformar el proceso de formulación del PND considerando evaluaciones ex ante basadas en evaluaciones de impacto ambiental para determinar las necesidades específicas, diseñar estrategias de desarrollo de la industria ambiental y satisfacer la demanda interna de BysA. Así se favorecería la construcción de ventajas comparativas que aumenten su competitividad en los mercados internacionales. Es necesario, además, fortalecer las capacidades domésticas para intervenir en el comercio internacional, pues los beneficios sociales, que son parte del desarrollo sostenible, han sido mucho menores que los beneficios privados. E1 Estado debe financiar diagnósticos ambientales, la investigación y el desarrollo para enfrentar las dificultades creadas por la apertura comercial (Bandeira, 2009).

\section{CONSIDERACIONES FINALES}

E1 TLCAN abrió el acceso a una mayor variedad de bienes ambientales. No obstante, la relación de México con los países socios, en especial con Estados Unidos, beneficia ante todo a la industria estadounidense. Además, sigue siendo preocupante la diversidad de factores que truncan la consolidación de la industria ambiental mexicana, como la falta de fondos de inversión públicos y privados, el tamaño y la capacidad de las empresas; los cuales exigen ir más allá de redefinir conceptos y listas de вysA para impulsar el comercio.

La nueva estrategia debe aprovechar las capacidades de las empresas pequeñas y medianas, sentar las condiciones para que aumenten su competitividad y reduzcan los costos de las metas de desarrollo sostenible, en el que se debe poner más empeño, e incluir las particularidades económicas, sociales y ambientales del país. La construcción de indicadores confiables, el Plan Nacional de Desarrollo y las evaluaciones de impacto ambiental pueden ayudar a detectar los factores contextuales de demanda de Bysa complementarios de las políticas sectoriales, para darles congruencia y determinar los bienes y servicios ambientales necesarios para cubrir la demanda interna. Una vez aumente el espíritu emprendedor y se aproveche la industria 
nacional con fines domésticos, se podrá revaluar el papel de acuerdos como el TLCAN y definir las ventajas comparativas necesarias para que compita en los mercados mundiales.

La investigación futura debería aportar estudios exhaustivos de los mecanismos de coordinación de las políticas económica y ambiental, para que a mediano plazo se reduzca la dependencia de tecnología importada para la gestión y protección del medio ambiente, sobre todo, de tecnología estadounidense.

\section{REFERENCIAS BIBLIOGRÁFICAS}

1. Ahumada, C.; Pelayo, B. et al. (2012). Sustentabilidad ambiental, del concepto a la práctica: una oportunidad para la implementación de la evaluación ambiental estratégica en México. Gestión y Politica Pública, 21(2), 451-483.

2. Avery, B. y Boadu O. F. (2004). Global demand for us environmental goods and services. Journal of Agricultural and Applied Economics, 36(1), 49-62.

3. Bandeira, P. (2009). Instituciones y desarrollo económico. Un marco conceptual. Revista de Economía Institucional, (13)25, 79-93.

4. Castrillón, A. (2013). Mercado y virtud o cómo complica la economía. A propósito de Las pasiones y los intereses, de Albert Hirschman, Revista de Economia Institucional, 11(20), 355-373.

5. Bojórquez, T. L.; Sánchez, C. S. et al. (2005). Building consensus in environmental impact assessment through multicriteria modeling and sensitivity analysis. Environmental Management, 36(3), 469-481.

6. Dutrénit, G.; Vera-Cruz, A. et al. (2003). Diferencias en el perfil de acumulación de capacidades tecnológicas de tres empresas mexicanas. El Trimestre Económico, 70(277), 109-162.

7. Comisión para la Cooperación Ambiental. (2004). Análisis del comercio de bienes y servicios ambientales en la región del TLCAN. [http://www3. cec.org/islandora/es/item/2142-examination-trade-in-environmentalgoods-and-services-in-nafta-region-es.pdf].

8. Feagans, B. (1997). Green lights. Business Mexico, 7(9), 38-42.

9. Ferrier, G. (2010). The evolution of the environmental industry in the post-NAFTa era in Mexico. International Environmental Agreements, 10, 147-164.

10. Gallegos, A. y Régibeau, P. (2008). Comercio estratégico y políticas ambientales para las industrias oligopólicas. El Trimestre Económico, $75(2), 317-345$.

11. González, S. (2008). Políticas e instituciones para el desarrollo económico territorial. El caso de México. Cepal, serie Comercio internacional, 2.

12. Guerrero, O. (1994). Los usos del análisis de implementación de políticas. Gestión y Política Pública, 3(1), 19-41.

13. Hanson, G. H. (2010). Why isn't Mexico rich? Journal of Economic Literature, 48(4), 987-1004.

14. Hamwey, R.; Hoffman, U. et al. (2003). Liberalization of international trade in environmental goods and services. Bangkok: UNCTAD. 
15. Kehoe, J. y Ruhl, J. (2011). ¿Por qué las reformas económicas de México no han generado crecimiento? El Trimestre Económico, 78(311), 491-518.

16. Konvitz, J. (2012). Infraestructura, deuda y desarrollo: lecciones actuales de la crisis mexicana de 1994-1996. Gestión y Politica Pública, 21(2), 451-483.

17. Lendo, E. (2005). Defining environmental goods and services: $A$ case study of Mexico. Ginebra: ICTsD.

18. Lindblom, C. E. (1994). La investigación social para la elaboración de políticas: quién la necesita y para qué. Gestión y Politica Pública, $3(2), 253-287$.

19. Lustig, N. (2001). Life is not easy: Mexico's quest for stability and growth. Journal of Economic Perspectives, 15(1), 85-106.

20. Mathew, A. y Fernández de C., S. (2009). The green dilemma about liberalization of trade in environmental goods. Journal of World Trade, 43(2), 379-416.

21. Morales, I. (1997). The Mexican crisis and the weakness of the NAFTA consensus. Annals of the American Academy of Political and Social Science, 550, 130-152.

22. Muñoz, V. C. (2005). Bienes y servicios ambientales en México: caracterización preliminar y sinergias entre protección ambiental, desarrollo del mercado y estrategia comercial. Cepal, serie Medio ambiente y desarrollo, 119.

23. ocDE. (1999). The environmental goods and services industry: Manual for data collection and analysis. Francia: Eurostat.

24. Perevochtchikova, M. (2013). La evaluación del impacto ambiental y la importancia de los indicadores ambientales. Gestión y Politica Pública, 22(2), 283-312.

25. Puyana, A. (2003). El camino mexicano hacia el regionalismo abierto: los acuerdos de libre comercio de México con América del Norte y Europa. Cepal, serie Comercio internacional, 35.

26. Ramírez, H. A.; Cadavid M. R. et al. (2011). Desempeño de las empresas y factores institucionales en Colombia, 2002-2007. Revista de Economía Institucional, 13(25), 179-198.

27. Ranger, M. E. (1993). The environment \& NAfTA. Business Mexico, 3(1), 78-79.

28. Sang, Y. H. y Jisun, K. (2011). Trade liberalization in environmental goods: Major issues and impacts. Korea and the World Economy, 12(3), 580-594.

29. Stern, A. M. (1993). A critique of eco-strategies. Business Mexico, 3(10), 40-43.

30. Tortajada, C. (2000). Environmental impact assessment of water projects in Mexico. International Journal of Water Resources Development, 16(1), 73-87.

31. Vikhlyaev, A. (2004). Environmental goods and services: Defining negotiations or negotiating definitions? Journal of World Trade, 38(1), 93-122.

32. Zhang, Z. (2010). Services the environment and the NAFta. International Environmental Agreements, 10, 89-91. 
33. Znamenacková, J.; Sauer, P. et al. (2014). Environmental goods market liberalization: Case study of Czech Republic and possible way to follow for Ukraine. Actual Problems of Economics, 151(1), 312-322. 